\title{
Götterdämmerung oder Morgenröte?
}

Peter Berchtold,

Christof Schmitz

Korrespondenz:

PD Dr. med. Peter Berchtold,

Dr. Christof Schmitz

College-M

Freiburgstrasse 41

CH-3010 Bern

Tel. 0316323026

Fax 0316323025

peter.berchtold@college-m.ch christof.schmitz@college-m.ch

www.college-m.ch
«Chefärzte an die kurze Leine». Schlagzeilen wie diese sorgten Anfang Juni dieses Jahres für Aufsehen. Konkret ging es um Vorschläge der Geschäftsprüfungskommission (GPK) des Zürcher Kantonsrates für eine Reorganisation des USZ. Einige Stimmen aus der Ärzteschaft meinten sodann, dass die Umsetzung dieser Vorschläge den Untergang des Universitätsspitals bedeuten würde, viele Stimmen in der Öffentlichkeit hingegen sympathisierten mit der Idee, dass Spitäler - oder sollte man sagen: Chefärzte? - mehr Management benötigen würden. Hintergrund dafür sind Empfindungen, dass sich in Spitälern zuwenig wandelt, dass zuwenig an «Kunden»im Sinne selbstverantwortlicher Patienten gedacht wird und dass ein Vielmehr an Reform möglich wäre. Während die Allgemeinheit mehr Management $\mathrm{zu}$ begrüssen scheint, wehren sich die Professionellen gegen einen Managerialismus, der einer Übersteuerung fachlicher Aufgaben durch fachfremde Manager bedeuten würde. Woran soll man sich halten? Wir wollen im Folgenden einen Beitrag zur Differenzierung dieser Debatte leisten.

Die Vorschläge der Zürcher GPK stehen im Kontext einer weit über Spitäler hinausreichenden Dynamik, nämlich der laufenden Weiterentwicklung sogenannter professioneller Organisationen. Im Kern geht es jeweils um die Frage, wie professionelles Handeln - z.B. des Arztes mit Managementhandeln kombiniert oder gar dominiert werden kann. Konkret für das USZ schlägt die GPK vor, dass der Chefarzt gemeinsam mit der Pflegeleitung und einem Klinikmanager eine sogenannte Klinikleitung bilden solle. Die Klinikmanager wären direkt der Spitaldirektorin unterstellt und würden analog der Spitalleitung den Vorsitz in der Klinikleitung einnehmen. Eine Idee, die in der Zwischenzeit im Zürcher Kantonsrat kontrovers diskutiert wurde und im Grundsatz schon länger in vielen Diskussionen insbesondere unter gepeinigten Spitaldirektoren und -direktorinnen vorkommt.

\section{Deutschland als Wegweiser?}

Wie bekannt, streikten in Deutschland heuer drei Monate lang die (Universitäts-)Klinikumsärzte. Bei diesem Arbeitskampf ging es vordergründig um Geld, dahinter aber standen und

\section{Crépuscule des dieux ou aube d'un jour nouveau?}

Comment peut-on réunir un savoir professionnel comme celui du médecin, avec celui de gestionnaire, p. ex. de clinique? Cette question préoccupe de plus en plus les milieux hospitaliers. Se fondant sur un rapport de la commission de gestion concernant la réorganisation de I'Hôpital universitaire de Zurich, l'article traite de ce thème et montre qu'il $y$ a des problèmes difficiles à résoudre sur le plan structurel. Agir professionnellement est la combinaison de connaissances individuelles, scientifiques et empiriques basées sur des cas, qui ne sauraient sinon être comprises et traitées de manière adéquate par personne d'autre. C'est du moins la perspective que l'on en a dans la profession médicale. Les médecins veulent éviter les mises en œuvre formelles et les hiérarchies étrangères à la discipline. Les gestionnaires de clinique sont néanmoins liés à des projets arrêtés, ainsi que de devoir montrer une éventuelle supériorité. Ils risquent ainsi de fortes réactions «anti-immunes» de médecins devant des propositions managériales. Il est d'autant plus important de viser l'intégration: savoir opérer la «reconnection» entre médecins et gestionnaires. Notre article tente de montrer comment la chose est possible.

stehen fundamentalere Probleme. Die Situation in Deutschland ist, wie aktuelle Forschungen eindrücklich zeigen, von einer Spannung zwischen Medizin und Management bzw. Ökonomie gekennzeichnet, die die Ärzte ins Mark trifft. Diese sehen ihr Verständnis dessen, was gute und sinnvolle Medizin bedeuten soll, immer schwieriger umsetzbar. Eine ganze Gilde zentraler Leistungsträger ist daran, die Identifikation mit ihren Institutionen zu verlieren. Das ist ein Alarmzeichen - auch für die Schweiz. Gleich- 
zeitig entwickeln die Ärzte Formen des Umgangs mit den neuen ökonomischen Anreizsystemen, die die gewünschten Wirkungen paradoxieren. Statt weniger Medizin gibt es mehr und intensivere, statt günstiger wird es teurer, statt koordinierter werden Patientenprozesse fragmentierter und statt besserer findet sich medizinische Qualität gefährdet [1].

Was läuft schief? Das Leben von Spitälern ist von vier stark voneinander differenzierten Subsystemen gekennzeichnet: der Medizin, der Pflege, dem Management sowie der Anbindung an die Öffentlichkeit via politische Gremien. Diese vier Subsysteme sind intensiv durch ihre je eigenen Kulturen, Denk- und Handlungsweisen bestimmt. Die besondere Schwierigkeit der Führwie der Reformierbarkeit von Spitälern ist wesentlich auf die spezielle Herausforderung der Integration dieser vier Subsysteme zurückzuführen, wie Sholom Glouberman und Henry Mintzberg ausgezeichnet beschrieben haben [2]. Das ist das Kreuz, das Spitäler zu tragen haben, und dieses gilt nicht nur für Zürich, sondern generell in Spitälern. Die Verschiedenheiten der Subsysteme beginnen jeweils sofort zu schwingen, wenn Einflusssphären neu geordnet werden. Da braucht es nicht viel, um das «Immunsystem» einer Profession zu aktivieren. Der Vorschlag der GPK, dass Chefärzte «ihre» Kliniken durch «Ökonomen» managen lassen sollen, rangiert auf einer diesbezüglichen Hitliste mindestens auf einem der ersten drei Plätze. Das heisst noch nicht, dass er schlecht ist. Allerdings lohnt es sich, präzise zu überlegen, was eine solche Führungsstruktur im Kontext einer Klinik bedeuten kann.

\section{Medizin ...}

Das Besondere des professionellen Handelns liegt in der je individuellen, fallbezogenen Kombination von wissenschaftlichem und Erfahrungswissen. Es ist der einzelne Arzt (seltener: ein Team), der dies vornimmt, denn es ist seine Aufgabe, wissenschaftlich legitimiert zu entscheiden und gleichzeitig der Einzigartigkeit des konkreten Patienten zu entsprechen. Diese Kombinationsleistung, gelegentlich mit der «Kunst des Arztes» (miss)bezeichnet, kann aus Sicht der Profession - von niemandem sonst adäquat verstanden, geschweige denn überprüft werden; nicht von anderen Spezialisten und schon gar nicht von Fachfremden wie Spitalleitern oder Klinikmanagern. Aus dieser Perspektive ergibt sich ein starker Anspruch auf kollegiale Selbstkontrolle sowie eine Tendenz zur Ablehnung formaler Vorgaben und von (fachfremder) Hierarchie: denn, «die haben keine Ahnung, wovon sie reden.» Auf formale Vorga- ben wären Klinikmanager aber angewiesen, denn erst mit solchen lassen sich jene Managementsysteme bauen, um die es aus dieser Sicht gehen muss. Und ebenso klar wären sie auf Akzeptanz ihrer Interventionen seitens der Ärzte angewiesen. Beides ist höchst voraussetzungsvoll. Die Erfahrung zeigt, dass Klinikmanager dann Akzeptanz finden, wenn sie Ärzte von «Administrativen» entlasten und sie mit ihrem Support die Fülle der betriebswirtschaftlichen Geschäfte, die Kliniken heute abarbeiten müssen, besser bewältigen lassen, aber keinen Anspruch und keine Kompetenz geltend machen, sich in das eigentliche Geschäft der Mediziner einzumischen.

Wobei die Grenzen zwischen Betriebswirtschaft und Medizin oft weniger klar zu ziehen sind, als man meinen könnte. Ärztliche Entscheidungen sind z.B. immer auch Ressourcenentscheidungen. Der Umfang der Diagnose, die Wahl des Medikaments, des Stents, des Entlassungstermins etc. sind immer auch Entscheidungen mit ökonomischen Konsequenzen. Fatal kann es werden, wenn die Constraints von aussen in einer Art und Weise von Fachfremden vorgegeben werden, die dazu führt, dass die Mediziner nicht mehr tun können, was sie meinen, medizinisch sinnvoll tun zu müssen. Auseinandersetzungen wie diese finden ihren Eingang mittlerweile sogar in Fernsehserien, wo Dr. House, der rüpelhafte, unführbare, aber beherzt und kompetent um das Wohl seiner Patienten kämpfende Chefarzt, beinahe dem potenten Aufsichtsratpräsidenten unterliegt. Aber nur beinahe... Gleichzeitig darf man fast beliebig annehmen, dass in den Prozessen der Spitäler noch erhebliche Optimierungspotentiale stecken, wie etwa neuere Anleihen bei Toyota, dem Aushängeschild der Automobilindustrie, zeigen. Schon mit dieser extrem groben Skizze wird deutlich, dass man sowohl Gründe für ein Primat der Medizin als auch für ein Mehr an Management anbringen kann. Und das eine schliesst das andere nicht aus.

\section{... und Management}

Wir halten fest: Management einer Klinik bedeutet, sich in einer Schnittmenge zwischen medizinischen, administrativen und eigentlich betriebswirtschaftlichen Aufgaben $\mathrm{zu}$ bewegen. Das macht es diffizil, das Management von Kliniken und eines Spitals zu organisieren und Strukturen zu finden, die passend differenzieren. Alle Versuche, strikte zu trennen, sind tendenziell zum Scheitern verurteilt, denn es geht um Komplementarität und nicht um Weisungsverhältnisse. In integrativen Approaches ist 
herauszufinden, welche Aufgaben- und Kompetenzmischungen angesichts einer speziellen Klinik- oder Spitalsituation adäquat sind. Zentrales Kriterium dafür ist das Gelingen eines multiperspektivischen Arbeitens zu den komplexen Entwicklungs- und Steuerungsfragen einer Klinik oder eines Spitals. Gutes Management zeichnet sich dann weniger durch Vorsitze aus denn durch das Gelingen, auch wider den Strich der bestehenden Verhältnisse gebürstete Qualität in Entscheidungsprozesse zu bringen und der Komplexität der Sache geschuldete Lösungen $\mathrm{zu}$ erarbeiten und umzusetzen. Das ist weniger durch Strukturen denn durch Prozesse zuwege zu bringen.

Professionelle Organisationen haben aus klassischer Managementsicht eine Schwäche. Sie verstehen sich als kollegial zu führende Organisationen. Entsprechend erwartbar weist der GPKBericht darauf hin, dass der ärztliche Direktor im USZ eher als Gleichrangiger denn als Vorgesetzter verstanden wird. Das überrascht keineswegs. Was stört allenfalls daran? Kann die Orientierung an Kollegialität etwas erschweren oder gar verunmöglichen? Wichtig ist hier: Kollegialität scheut tendenziell vor Konflikten eher zurück. Dann kreist man in der Chefärztekonferenz, sich wechselseitig beobachtend, unentschieden um sich selbst - und wird dann von externen Entwicklungen überrascht. Nicht umsonst kommen Reformschritte in Spitälern oftmals per Entscheid von aussen und nicht von innen her zustande. Oder aber, die Titanen stürzen sich aufeinander und zelebrieren sich in machtvollen Richtungskämpfen - und kommen auch so nicht voran. Zwischen dieser Scylla und Charybdis navigieren solche Organisationen. Sie versuchen, die Besonderheiten ihres professionellen Handelns zu bewahren und gleichzeitig ihre Organisation steuerbar $\mathrm{zu}$ halten. Heute heisst das wesentlich, sich strategisch verbindlich zu positionieren und sich stärker als anhin ökonomischen Zielen und Agenden zu unterwerfen. Das erfordert mehr Management denn je, und damit sind wir wieder beim Kern der Sache. Wie lassen sich professionelles und Managementhandeln kombinieren oder integrieren?

\section{Integriert?}

Oft genug tendiert man heute dazu, die Profession mit ökonomischen Anreizsystemen zu übersteuern. Etliche dieser Interventionen drohen die Zustände zu verschlechtern, statt zu verbessern. Eben darauf scheint das deutsche Beispiel im Moment hinauszulaufen, auch wenn es noch zu früh ist, ein endgültiges Urteil zu sprechen. Als unbeabsichtigte Nebenfolge wird neben den beschriebenen paradoxen Effekten die Identifikation der Professionellen, die Qualität und Leistung hervorbringen sollen, gefährdet. (Hoch)Leistung erfolgt nur dann, wenn die Ziele und Werte der relevanten Akteure mit den Rahmenbedingungen einigermassen stimmig sind. Alignment heisst das Stichwort dazu. Dieses erscheint heute vielfach gefährdet [3].

Die Vorschläge der GPK können in diesem Sinne die Konfrontation zwischen Managementund Professionslogik zuspitzen. Dem Ungenügen (chef-)ärztlicher Führung soll betriebswirtschaftliche Steuerungskompetenz zur Seite gestellt werden. Vielleicht ist das aktuell hilfreich, um Entscheidungen anzuregen. Insgesamt geht es um einen Approach, der sich der Differenzen und der konkreten Aufgabendynamiken im Spital und seinen Kliniken bewusst ist und kontinuierlich an Lösungen arbeitet, die die verschiedenen Logiken sinnvoll und pragmatisch miteinander integrieren lassen. Führungsrealitäten in Spitälern werden in komplexen, vernetzten Führungsabläufen gelebt, die weder durch einfache Strukturierungen noch durch heftiges Alpha-TierBrustklopfen bestimmt werden können. An und mit diesen Komplexitäten ist zu arbeiten. Die unterschiedlichen Kerngeschäfte Patientenbetreuung, Bildung und Forschung verlangen eine integrierende Behandlung ihrer je eigenen Zielsetzungen und Logiken. Gerade weil kein Mensch der beste Operateur, der beste Lehrer, der beste Forscher, die beste Führungsperson und der beste Controller sein kann - was selbst für medizinische Ordinarien mit MBA-Abschluss gilt -, lebt ein Spital davon, dass diese Bereiche in engem Bezug zueinander gestaltet werden. Dies gelingt heute nur noch durch integrative $\mathrm{Zu}$ gänge und Stärkung der Kooperation zwischen und mit den Professionisten und Subsystemen. Reconnecting lautet das Stichwort hierzu. Führung und Führungspersonen gleich welcher Couleurs haben die prioritäre Aufgabe, diese Kooperation zu fördern und diese zu betreiben, und zwar ohne kompromisslerische Abstriche bei der Qualität der Lösungen hinzunehmen.

\section{Ganz anders?}

Passung der Steuerung zu den Aufgaben und Dynamiken heisst auch, dass noch ganz andere Formen als Klinikmanager zu überlegen wären. In der Funktion des Klinikdirektors kulminiert heute tatsächlich ein Bündel von Ansprüchen, das höchst anspruchsvoll zu bewältigen ist. Die Differenzierung etwa von Dienstleistungsund Forschungsaufgaben, die mit Einführung der Spitalfachärzte auf den Ebenen unter den Chefärzten vorgenommen wurde, könnte auch 
auf Chefarztebene eine interessante Alternative darstellen. Ohne hier ins Detail gehen zu wollen, das Feld der Lösungen wäre jedenfalls zu erweitern.

Weitere wichtige Optionen bilden Formen wie Zentren, Departemente oder Bereiche, die allesamt Versuche darstellen, klinikübergreifende Momente zum Tragen zu bringen. Diese können sowohl aus medizinischer Sicht als auch aus Gesamtsteuerungsperspektive relevant sein. Ohnehin steht bei letzterer weniger das Management der einzelnen Klinik im Vordergrund, als die Frage: wie führt man z.B. 20-42 Kliniken? Kein Unternehmen gönnt sich eine derartige Führungsspanne - wohlweislich. Die Frage der Bündelung von Kliniken oder Leistungen von Kliniken stellt sich damit um so intensiver. Die Crux liegt dabei darin, Formen zu finden, die medizinisch wie organisatorisch tatsächlich Nutzen stiften können. Ist das der Fall, haben Spitäler so etwas wie strategische Geschäftseinheiten gewonnen, die für sich Steuerbarkeit in Anspruch nehmen können. Die Leitungsfrage ist dann natürlich hochinteressant - und gibt wieder zu diskutieren: soll das ein Arzt oder ein Manager sein...

Die Antwort auf diese Frage - will man dazu die Erfahrung aus anderen Unternehmen nutzen - kann nur heissen Mediziner, und zwar darum, weil das Beste, was wir für komplexe Führungsaufgaben haben können, Menschen mit sogenannten A-Skills sind. Das sind solche, die über echte Kompetenz in zwei Fachgebieten verfügen. Nachdem es den wenigsten Managern gelingen dürfte, sich ausreichend in Medizin nebenbei zu spezialisieren, bieten sich die Ärzte doppelt an. Werden die Ärzte diese Herausforderung annehmen? Und: wer wird das akzeptieren?

\section{Literatur}

1 Vogd W. Die Organisation Krankenhaus im Wandel. Bern: Huber; 2006.

2 Glouberman S, Mintzberg H. Managing the care of health and the cure of disease - Part I: Differentiation. Health Care Manage Rev. 2001;26(1):56-92.

3 Gardner H, Csikszentmihalyi M, Damon W. Good Work! Wiesbaden: Klett-Cotta; 2005. 